\title{
Vibrational properties and stability of FePt nanoalloys
}

\author{
Przemysław Piekarz, Jan Łażewski, Paweł T. Jochym, Małgorzata Sternik, and Krzysztof Parlinski \\ Institute of Nuclear Physics, Polish Academy of Sciences, Radzikowskiego 152, PL-31342 Kraków, Poland
}

(Dated: November 13, 2018)

\begin{abstract}
The structural and dynamical properties of FePt nanoparticles were studied within the density functional theory. The effect of size and chemical composition on dynamical stability of nanoparticles was investigated for the cuboctahedral and icosahedral symmetries. In cuboctahedra, the structural distortion is observed, which for systems with odd number of Pt layers leads to lowering of the tetragonal symmetry. Significant differences between the vibrational properties of FePt particles and bulk crystal is observed, but similarly to the FePt crystal, cuboctahedral particles exhibit a strong anisotropy of atomic vibrations. The icosahedral particles with perfect shell geometry are unstable due to enlarged distances between Fe atoms. They can be stabilized by removing a central atom or replacing it by a smaller one. The heat capacity and entropy of nanoparticles show typical enhancement due to low-energy vibrations at the surface layers.

PACS numbers: 63.20.kn, 63.20.dk, 65.40.Ba, 65.80.-g
\end{abstract}

\section{INTRODUCTION}

Distinct properties of nanoparticles (NPs) stem from their intermediate size between molecular systems and crystals, sharing features with both of these groups. Nanoparticle alloys (nanoalloys) are bi- or multicomponent metallic particles, often with the complex structures and properties, which can be very different from those of the corresponding bulk alloys and single-metal nanoparticles [1-3]. By adjusting the size and chemical composition, nanoalloys can be optimized for the applications in catalysis [4, nanomedicine [5, and data storage [6].

Dynamical properties of nanoobjects are significantly modified comparing to bulk systems due to reduced dimensionality and surface effects $7+22$. The theoretical studies on vibrational properties of isolated NPs, performed with the empirical potentials [10-13] and density functional theory (DFT) [18, 19], demonstrated the pronounced changes in the vibrational density of states (VDOS) comparing to bulk systems. The increase of the VDOS at low energies is caused mainly by the surface atoms with the reduced coordination number, while the shift of the energies to higher values above the bulk cutoff is induced by the inner atoms with shorter interatomic distances [10, 11]. Vibrational properties of a NP depend substantially on its size and geometry [19], as well as on the presence of adsorbate atoms 18. In nanoalloys, dynamics of atoms depends additionally on local environment and atomic coordination [23. These changes influence thermodynamical properties such as thermal expansion, heat capacity, melting temperature, and thermal displacements and make the standard approaches, like Debye model, not applicable for NPs [19, 24.

Experimentally, the effect of reduced size on vibrational properties of NPs have been studied using the nuclear inelastic scattering (NIS) 25 27 and the optical methods such as multiple photon dissociation spectroscopy in the far-infrared [28, time-resolved pumpprobe spectroscopy [29, 30, and Raman scattering [3134. For instance, the energies of the lowest modes (acoustic gap) and the symmetric modes inducing the radial expansion and contraction of particles (breathing mode) can be obtained from the pump-probe experiments [29, 30].

Iron-platinum (FePt) alloys, crystallizing in the layered $L 1_{0}$ phase, exhibit an extremely high uniaxial magnetocrystalline anisotropy [35. FePt is therefore a promising material for applications in ultra-dense magnetic recording media provided that well-ordered $L 1_{0}$-particles of small size can be synthesized [6, 36 38 . Relevant information about the stability and electronic properties of the FePt nanoclusters were obtained by theoretical studies 39. The Monte Carlo simulations show that melting temperatures of FePt NPs are much lower than the bulk phase-transition temperature $(1572 \mathrm{~K})$ and decrease with the particle size reduction [40]. The structural order and magnetic properties of $\mathrm{FePt}$ nanoalloys with various morphologies were studied previously within the DFT calculations 41 44. Recently, we have investigated the influence of chemical composition on the stability of FePt icosahedra using the ab initio molecular dynamics (MD) simulations [45.

The structural anisotropy influences also the dynamical properties of the FePt systems. The partial Fe VDOS obtained in the composite of FePt NPs [4], showed a large difference between the spectra measured parallel and perpendicular to the $c$ axis. Similar anisotropy was found in the thin films studied experimentally [47] and by the ab initio calculations performed for the bulk FePt crystal [46 48. These studies revealed also differences between the spectra of NPs and thin films, mainly at low energies, which result from the surface effects and/or phonon confinement. It has been suggested that the excess of the low-energy modes in the Fe partial DOS may indicate the appearance of the Fe-terminated surface in the NPs [47. Also, another interesting question arises: How the dynamical anisotropy depends on the size and geometry, and how it affects the thermodynamical properties of FePt nanoalloys?

In this paper, we investigate the structural and dy- 
namical properties of selected FePt nanoparticles with the cuboctahedral and icosahedral geometries using the first-principles DFT methods. Comparing to the semiempirical potentials, the $a b$ initio approach is limited to small nanoparticles, but it explicitly includes the effect of electronic structure and magnetic interactions on atomic forces and dynamical properties of particles. We study the effects of a size and chemical composition on a structural stability and vibrational spectra of NPs and analyze the structural deformations induced by the soft modes. Finally, using the vibrational spectra, we calculate the heat capacity and entropy of nanoalloys as functions of temperature and system size.

The paper is organized as follows. In Sec. II, the calculation method used in the studies of structural and dynamical properties of FePt NPs is presented. Sec. IIIa and IIIb describe the results of calculations for the cuboctahedral and icosahedral particles, respectively. In Sec. $\mathrm{IV}$, the thermodynamic properties of nanoparticles are presented and discussed. Sec.V summarizes presented results.

\section{CALCULATION METHOD}

The calculations were performed within the DFT as implemented in the Vienna Ab initio Simulation Package (VASP) [49. The exchange-correlation functionals are described by the generalized-gradient approximation (GGA) [50] within the projector augmented-wave method [51]. The calculations were performed in periodic boundary conditions in a cubic supercell of properly chosen size $d$ to keep the distance between a particle and its images larger than $12 \AA$, large enough to exclude long-range interactions. All reciprocal-space summations are performed only at the $\Gamma$ point. The valence states where optimized with the Fe $3 d^{6} 4 s^{2}$ and $\mathrm{Pt} 5 d^{9} 6 s^{1}$ electron configurations. The first-order Methfessel-Paxton scheme was applied for the Fermi surface smearing with $\sigma=0.2 \mathrm{eV}$. The cut-off energy of $320 \mathrm{eV}$ was used for the plane-wave expansion. The convergence criteria were set to $10^{-7} \mathrm{eV}$ and $10^{-4} \mathrm{eV} / \AA$ for the total energy and residual forces, respectively.

The calculations for the $L 1_{0}-\mathrm{FePt}$ crystal with the space group $P 4 / \mathrm{mmm}$ were performed in the $2 \times 2 \times 2$ supercell containing 32 atoms. The wave functions were sampled over the points generated with Monkhorst-Pack scheme using the $4 \times 4 \times 4 \mathbf{k}$-point grid. In all calculations we assumed the ferromagnetic (FM) order on $\mathrm{Fe}$ atoms, which is an experimentally observed state in the FePt crystal and nanoalloys.

We have chosen two basic morphologies of NPs: the cuboctahedron with the point group symmetry $D_{4 h}$ and the icosahedron $\left(I_{h}\right)$ presented in Fig. 1. The ideal cuboctahedron is a piece of the tetragonal FePt crystal $\left(L 1_{0}\right)$. With the imposed periodic boundary conditions not all symmetry elements of the icosahedra are taken into account, and the relaxation has been performed with the
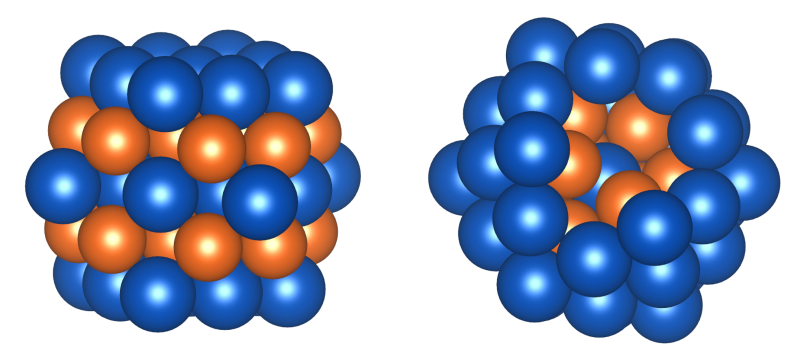

FIG. 1. Side view of the cuboctahedral $\mathrm{Fe}_{24} \mathrm{Pt}_{31}$ (left) and icosahedral $\mathrm{Fe}_{12} \mathrm{Pt}_{43}$ (right) nanoparticle. Pt atoms are in blue (dark) and Fe atoms in red (light) colors. For the icosahedral nanoparticle a few atoms from the outer layers were removed to show its alternating shells structure. The drawing prepared with the VESTA software [59].

lower point group $T_{h}$. Nevertheless, due to large enough supercells all group elements of the $I_{h}$ symmetry, including the fivefold rotation axis $C_{5}$, are preserved with the accuracy better than $10^{-3} \AA$. We consider the clusters with the magic numbers of atoms $N$ defined by the following formula:

$$
N=\left(10 n^{3}+15 n^{2}+11 n+3\right) / 3=13,55,147, \ldots
$$

where $n$ is the number of closed shells. Additionally, the icosahedral particles without the central atom with $\mathrm{N}=12$ and 54, and the core-shell $\mathrm{Fe}_{13} \mathrm{Pt}_{42}$ system where studied. For selected NPs, we have swapped Fe and Pt to investigate the influence of atomic configuration on structural and dynamical properties. All studied systems are listed in Tab. I.

Vibrational energies and polarization vectors were derived using the direct method [52] implemented in the

TABLE I. The list of studied nanoparticles with defined size, symmetry, and chemical composition.

\begin{tabular}{lccc}
\hline$N$ System & Symmetry & Soft modes & Ground state \\
\hline $13 \mathrm{Fe}_{5} \mathrm{Pt}_{8}$ & $D_{4 h}$ & $A_{1 g}, B_{1 g}$ & $C_{1}$ \\
$13 \mathrm{Fe}_{8} \mathrm{Pt}_{5}$ & $D_{4 h}$ & $B_{2 u}, B_{1 g}$ & - \\
$13 \mathrm{FePt}_{12}$ & $I_{h}\left(T_{h}\right)$ & No & $I_{h}\left(T_{h}\right)$ \\
$13 \mathrm{Pt}_{13}$ & $I_{h}\left(T_{h}\right)$ & No & $I_{h}\left(T_{h}\right)$ \\
$13 \mathrm{Fe}_{12} \mathrm{Pt}$ & $I_{h}\left(T_{h}\right)$ & $2 T_{u}, E_{u}, A_{g}, T_{g}, A_{u}$ & - \\
$12 \mathrm{Fe}_{12}$ & $I_{h}\left(T_{h}\right)$ & No & $I_{h}\left(T_{h}\right)$ \\
\hline $55 \mathrm{Fe}_{24} \mathrm{Pt}_{31}$ & $D_{4 h}$ & $A_{2 u}$ & $C_{4 v}$ \\
$55 \mathrm{Fe}_{31} \mathrm{Pt}_{24}$ & $D_{4 h}$ & $B_{1 g}, A_{2 g}, E_{u}$ & - \\
$55 \mathrm{Fe}_{12} \mathrm{Pt}_{43}$ & $I_{h}\left(T_{h}\right)$ & $E_{g}, T_{g}$ & - \\
$54 \mathrm{Fe}_{12} \mathrm{Pt}_{42}$ & $I_{h}\left(T_{h}\right)$ & No & $I_{h}\left(T_{h}\right)$ \\
$55 \mathrm{Fe}_{13} \mathrm{Pt}_{42}$ & $I_{h}\left(T_{h}\right)$ & No & $I_{h}\left(T_{h}\right)$ \\
$55 \mathrm{Fe}_{43} \mathrm{Pt}_{12}$ & $I_{h}\left(T_{h}\right)$ & $A_{g}, E_{u}, T_{u}$ & - \\
$54 \mathrm{Fe}_{42} \mathrm{Pt}_{12}$ & $I_{h}\left(T_{h}\right)$ & No & $I_{h}\left(T_{h}\right)$ \\
\hline $147 \mathrm{Fe}_{67} \mathrm{Pt}_{80}$ & $D_{4 h}$ & No & $D_{4 h}$ \\
$147 \mathrm{Fe}_{80} \mathrm{Pt}_{67}$ & $D_{4 h}$ & $A_{1 u}, E_{g}, B_{1 u}, B_{1 g}$ & - \\
\hline
\end{tabular}


PHONON program [53]. First, we optimize the system to obtain the positions of all atoms for the assumed symmetry. Next, the Hellmann-Feynman (HF) forces are calculated by displacing atoms from the equilibrium positions, one at a time, in positive and negative directions by $0.03 \AA$. The number of required displacements is determined by the number of non-equivalent atoms and atom site symmetries. From the HF forces obtained with respective displacements, the force-constants are calculated and dynamical matrices are constructed. Finally, the vibrational energies and polarization vectors are extracted by diagonalization of the dynamical matrix at the $\Gamma$ point. The vibrational spectra of small NPs consist of separate lines, which are broadened using Gaussian functions with the width $1 \mathrm{meV}$ FWHM for better presentation.

The obtained vibrational spectrum can be used to study the stability of the system optimized in the assumed geometry. If all eigenvalues are real, the system is dynamically stable and there is no additional deformation of the particle. The presence of imaginary energies in a spectrum indicates a possible structural deformation, which results in lowering of the symmetry. Polarization vectors of soft modes give complete information of atomic displacements from high-symmetry positions towards a more stable system with the lower symmetry.

The described calculation procedure has two main advantages. By using the higher symmetry of particles in the first step, the computational time can be reduced comparing to calculations without symmetry constraints, and larger systems can be studied in a reasonable time. More important advantage is a possibility to reveal the phonon modes, which are responsible for structural deformations of particles. As we discuss below, such soft modes exist in some NPs and lead to configurations with lower symmetries. For the stable systems, the vibrational heat capacities and entropies are calculated within the harmonic approximation.

\section{VIBRATIONAL PROPERTIES}

\section{A. Cuboctahedra}

A cuboctahedral NP can be treated as a piece of the bulk, therefore, the vibrational properties discussed in this section will be compared with the results obtained for the FePt crystal [48. First, we recall the main features of the phonon DOS for this system presented in Fig. 2. The whole spectrum can be divided into two regions: lowenergy part (below approx. $20 \mathrm{meV}$ ), where vibrations of $\mathrm{Pt}$ atoms dominate and high-energy region with the main contribution from Fe atoms. The partial DOS functions projected along the $x y$ and $z$ directions demonstrate a large anisotropy of lattice dynamics in this structure [48]. The lowest band $(8-12 \mathrm{meV})$, consists mainly of movement of $\mathrm{Pt}$ atoms along the $z$ direction. Energies of $\mathrm{Pt}$ vibrations in $x y$ directions are distributed over al-

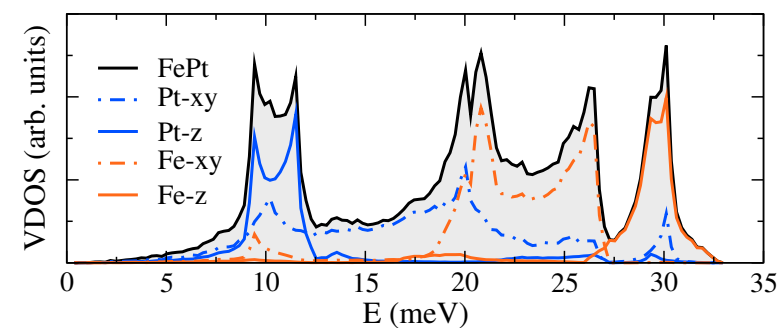

FIG. 2. Phonon density of states calculated for the FePt crystal in $P 4 / \mathrm{mmm}$ symmetry $\left(L 1_{0}\right)$.

most the entire spectrum with a maximum at $20 \mathrm{meV}$. Vibrations of Fe atoms mainly contribute to two energy regions: $18-28 \mathrm{meV}(x y)$ and $26-33 \mathrm{meV}(z)$.

$\mathbf{F e}_{\mathbf{5}} \mathbf{P t}_{\mathbf{8}}-$ The smallest considered particle consists of 13 atoms arranged in two Pt layers of four atoms each, separated by a single Fe layer of five atoms (see Fig. 3). This particle was optimized in the cubic supercell with size $d=20 \AA$, which gives the distance between the particle and its nearest image equal to $16 \AA$. The obtained distance between $\mathrm{Fe}$ and Pt layers equals $1.75 \AA$, so it is reduced with respect to the bulk value of $1.86 \AA$. Comparing to the bulk lattice parameter $a=3.85 \AA$, the corresponding $\mathrm{Fe}-\mathrm{Fe}$ and $\mathrm{Pt}-\mathrm{Pt}$ distances are equal 3.48 and $3.92 \AA$, respectively. In the vibrational spectrum, shown in Fig. 4 , there are 33 modes $(3 N-6)$. The low-energy part, where $\mathrm{Pt}$ vibrations dominate, is separated by a gap (13-17 meV) from the high-energy part of mainly Fe vibrations. Performing calculations within the tetragonal $D_{4 h}$ symmetry we found two soft modes with the $B_{1 g}$ and $A_{1 g}$ symmetries and the very close imaginary energies $4.45 i \mathrm{meV}$ and $4.39 i \mathrm{meV}$, respectively. Using the standard convention these imaginary modes are plotted with negative energies [see Fig. 4(a)]. The fully symmetric mode $A_{1 g}$ does not change the symmetry. In the $B_{1 g}$ mode, two Pt atoms move outward and two inward (see

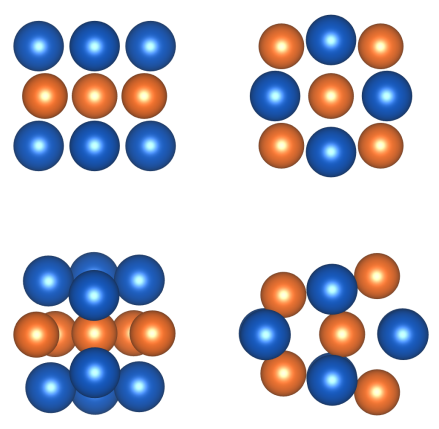

FIG. 3. Side (left column) and top view (right column) of the cuboctahedral $\mathrm{Fe}_{5} \mathrm{Pt}_{8}$ particle with $D_{4 h}$ (top row) and $C_{1}$ symmetry (bottom row). Pt atoms are in blue (dark) and Fe atoms in red (light) colors. The atomic radii were reduced accordingly to reveal displacements of atoms. 


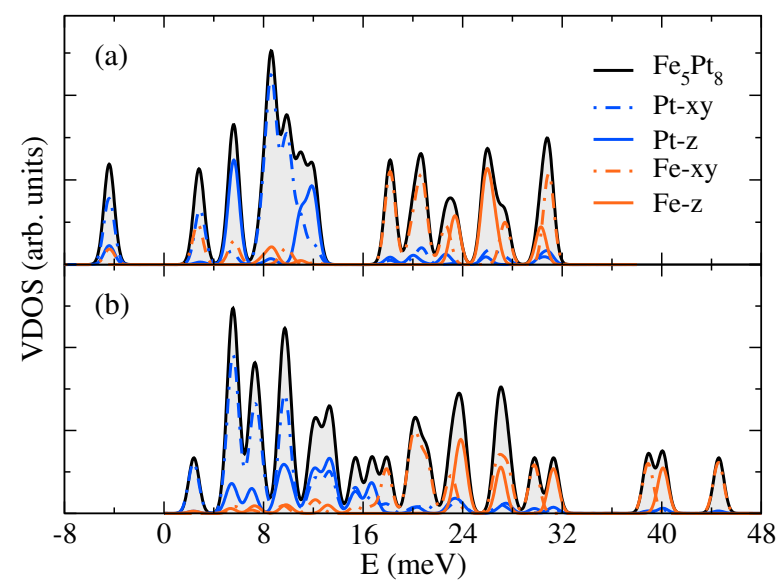

FIG. 4. Vibrational density of states of the $\mathrm{Fe}_{5} \mathrm{Pt}_{8}$ cuboctahedron calculated for (a) $D_{4 h}$ and (b) $C_{1}$ structures.

the animation in Supplemental Material [54]), distorting asymmetrically the particle and lowering its symmetry to $D_{2 h}$. The cluster optimized in this symmetry has lower total energy (by $32 \mathrm{meV} /$ atom). However, this structure is still unstable and after final optimization without symmetry elements $\left(C_{1}\right)$, we get further lowering of the energy (by $15 \mathrm{meV} /$ atom). The final geometry of the particle is compared with the perfect cuboctahedron in Fig. 3. It has asymmetric shape, elongated in one direction and compressed in another. Some interatomic distances are strongly reduced, some of them enlarged. The origin of such asymmetric deformation can be related with the changes in the electronic structure. It removes the spin-down (minority) states from the Fermi energy and splits the spin-up states, partially removing its degeneracy, similarly to the Jahn-Teller effect (see Supplemental Material [54]).

The resulting vibrational spectrum consists of only real energies confirming the dynamic stability of the obtained geometry [see Fig. 4(b)]. Deformation of the particle causes large changes in the VDOS. Symmetry lowering splits the degenerate modes and leads to effective broadening of the low- and high-energy bands as well as closing the gap. More interesting is appearance of the vibrational states at much higher energies (above $38 \mathrm{meV}$ ), even comparing to the phonon energies in the iron bcc crystal [55. These vibrations originate from only one $\mathrm{Fe}$ atom located in the center of the particle, and the highest mode at $44.6 \mathrm{meV}$ corresponds to the in-plane vibration of this atom. Such high energies are induced by the Fe-Fe distance $(2.36 \AA)$, strongly reduced in comparison to the undistorted system $(2.46 \AA)$. Also, the distances between the central atom and three $\mathrm{Pt}$ atoms are reduced in the distorted particle.

$\mathbf{F e}_{\mathbf{8}} \mathbf{P t}_{\mathbf{5}}$ - The nanoparticle $\mathrm{Fe}_{8} \mathrm{Pt}_{5}$ consists of one inner Pt layer with five atoms and two surface Fe layers with four atoms each. The distance between nearest layers $(1.86 \AA)$ equals to the bulk value. All interatomic distances are reduced comparing to the $\mathrm{Fe}_{5} \mathrm{Pt}_{8}$ nanoparticle

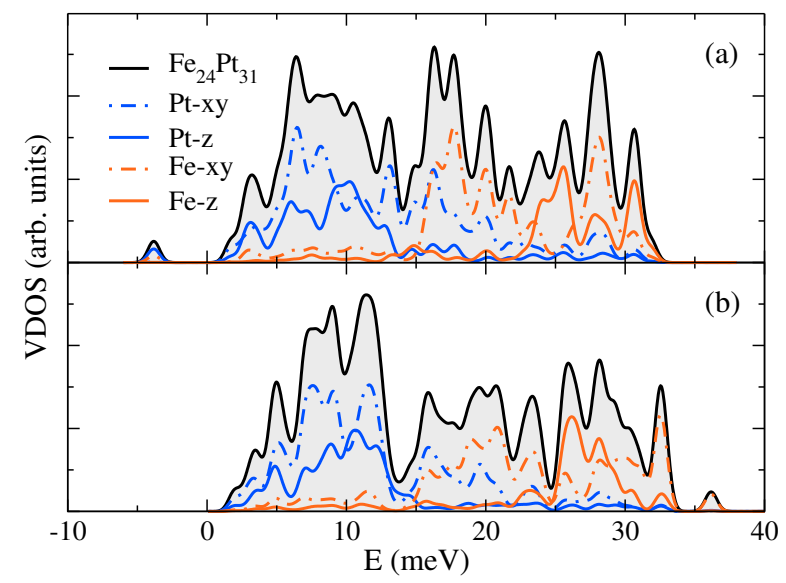

FIG. 5. VDOS of the $\mathrm{Fe}_{24} \mathrm{Pt}_{31}$ cuboctahedron calculated for (a) $D_{4 h}$ and (b) $C_{4 v}$ structures.

and the bulk FePt crystal. This compressed geometry is very unstable and generates two soft modes with the symmetries $B_{1 g}$ and $B_{2 u}$ (see Tab. II). The polarization vectors were used to distort the particle, which relaxes to the lower symmetry $\left(C_{2 v}\right)$ with the reduced total energy by $38 \mathrm{meV} /$ atom. Additional relaxation of the system without the symmetry constraints leads to further lowering of the total energy by $6 \mathrm{meV} /$ atom. The final particle geometry is characterized by a strong deformation of the Fe layers and it is rather metastable, dynamically unstable configuration of atoms. There are still soft modes in the resulting vibrational spectrum (not shown).

$\mathbf{F e}_{\mathbf{2 4}} \mathbf{P t}_{\mathbf{3 1}}-$ The next system we consider is the cuboctahedron with 55 atoms. It is built of $3 \mathrm{Pt}$ and $2 \mathrm{Fe}$ layers, with slightly elongated outer interlayer distance $(1.88 \AA)$ and shortened inner distance $(1.80 \AA)$ comparing to the bulk value $(1.86 \AA)$. The obtained total and partial vibrational spectra projected along the $x y$ and $z$ directions are presented in Fig. 5. The differences between these projections show anisotropy in atomic vibrations. Similarly to the phonon DOS of the FePt crystal, the highenergy region is dominated by Fe vibrations along the $z$ direction, and low-energy part by $\mathrm{Pt}$ atoms vibrating in the $x y$ plane. The energy cut-off is only slightly shifted above the bulk limit $(32 \mathrm{meV})$. In contrast, the density of states largely increases in the range below $4 \mathrm{meV}$, with main contribution from platinum surface atoms. Additionally, there is one soft mode $A_{2 u}$ with the energy of $3.83 i \mathrm{meV}$, which reduces the symmetry $D_{4 h}$ to its subgroup $C_{4 v}$. In this soft mode, the Fe atoms move in $x y$ and $\mathrm{Pt}$ atoms in $z$ directions, and only the surface atoms are involved (see Supplemental Material [54). After optimization of the system distorted by this mode, the total energy decreases by about $7 \mathrm{meV} /$ atom only. We verified also that additional optimization without symmetry constraints does not lower the total energy. It means that the structure obtained within the $C_{4 v}$ symmetry is the ground state of the $\mathrm{Fe}_{24} \mathrm{Pt}_{31}$ cuboctahedron. The structures with the $D_{4 h}$ and $C_{4 v}$ symmetries are com- 

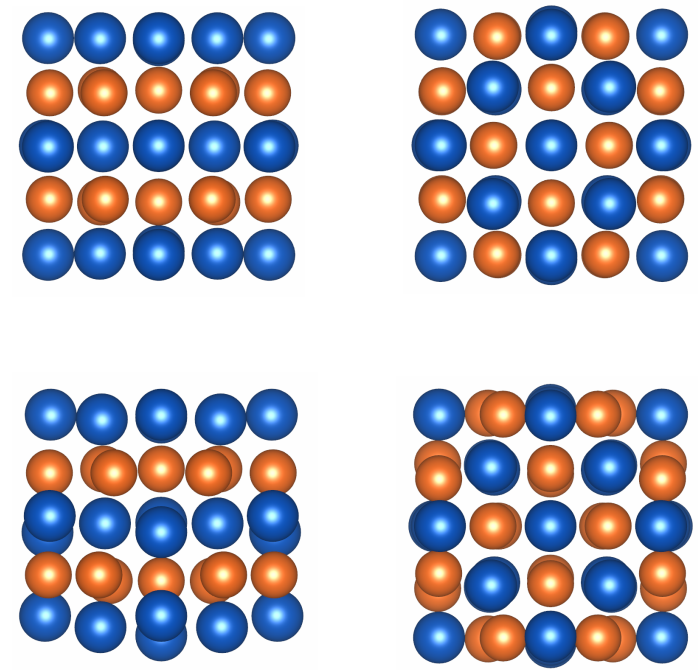

FIG. 6. Side (left column) and top view (right column) of the cuboctahedral $\mathrm{Fe}_{24} \mathrm{Pt}_{31}$ particle with $D_{4 h}$ (top row) and $C_{4 v}$ symmetry (bottom row). Pt atoms are in blue (dark) and Fe atoms in red (light) colors.

pared in Fig. 6. In the distorted structure, some Fe-Fe and $\mathrm{Pt}-\mathrm{Pt}$ distances are reduced along the $x y$ and $z$ directions, respectively. Such dimerization was found for the first time in calculations for larger FePt nanoparticles [42]. Our study reveals the mechanism of deformation, in which the soft mode brakes the $D_{4 h}$ symmetry of perfect cuboctahedral particles. The VDOS calculated for the distorted particle does not contain any soft mode [see Fig. 5(b)], however, there are substantial changes observed mainly at high energies. The energy cut-off is now shifted above $36 \mathrm{meV}$, and surprisingly, the highest mode corresponds to the in-plane $(x y)$ Fe vibrations.

$\mathbf{F e}_{\mathbf{3 1}} \mathbf{P t}_{\mathbf{2 4}}-$ We have studied also the system with the exchanged $\mathrm{Fe}$ and $\mathrm{Pt}$ atoms. Having $\mathrm{Fe}$ atoms in the upper and bottom surface layers, this system is very unstable. In the vibrational spectrum, there are three soft modes with the symmetries $B_{1 g}, A_{2 g}$, and $E_{u}$, which all together strongly distort this NP lowering its symmetry to $C_{1}$. After relaxation of the distorted particle, the total energy decreases by $51 \mathrm{meV} /$ atom but there are still three soft modes and the system is dynamically unstable.

$\mathbf{F e}_{\mathbf{6 7}} \mathbf{P t}_{\mathbf{8 0}}-$ The cuboctahedron with $N=147$ consists of $4 \mathrm{Pt}$ and $3 \mathrm{Fe}$ layers. For this system, we have increased the linear size of the supercell to $d=25 \AA$, to keep the distance between the particle and its images larger than $13 \AA$. The optimized particle is presented in Fig. 7. Interestingly, it shows the same distortion (dimerization) as observed in Fig. 6 and discussed in Ref. [42]. In the obtained VDOS presented in Fig. 8, there are no soft modes indicating that the structure is dynamically stable. In this case, the $D_{4 h}$ symmetry is not broken and the horizontal mirror plane is preserved. Thus, we can state a general rule that the FePt cuboctahedral NPs

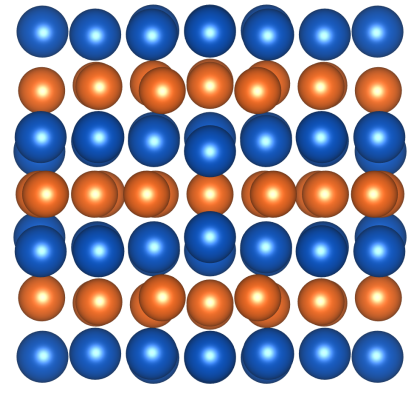

FIG. 7. Side view of the cuboctahedral $\mathrm{Fe}_{67} \mathrm{Pt}_{80}$ particle with $D_{4 h}$ symmetry. Pt atoms are in blue (dark) and Fe atoms in red (light) colors.

with an even number of $\mathrm{Pt}$ layers shows the distortion, which preserves the $D_{4 h}$ symmetry and in those with an odd number of Pt layers, the symmetry is lowered by the soft-mode mechanism: $D_{4 h} \rightarrow A_{2 u} \rightarrow C_{4 v}$. The vibrational spectrum of this larger particle is more anisotropic than in the smaller one. The $z$-projected VDOS is shifted to the highest energies as in the bulk spectrum, however, the energy cut-off is smaller than in the FePt crystal. Similarly to smaller particles, there is an increase of the low-energy states coming from the Pt surface atoms.

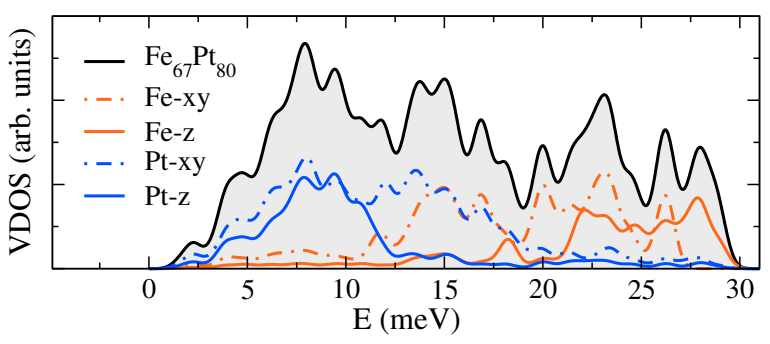

FIG. 8. VDOS of the $\mathrm{Fe}_{67} \mathrm{Pt}_{80}$ cuboctahedron.

$\mathbf{F e}_{\mathbf{8 0}} \mathbf{P t}_{\mathbf{6 7}}-$ Similarly to the smaller systems, the nanoparticle $\mathrm{Fe}_{80} \mathrm{Pt}_{67}$ with the Fe atoms in the surface layers is also very unstable. There are four soft modes listed in Tab. If We have not studied this nanoparticle any further.

\section{B. Icosahedra}

$\mathbf{F e P t}_{12}, \mathbf{F e}_{12} \mathbf{P t}-$ The smallest icosahedron consists of one central atom $\mathrm{Fe}(\mathrm{Pt})$ and twelve $\mathrm{Pt}(\mathrm{Fe})$ atoms placed at equal distances from the center. This distance is larger in the $\mathrm{FePt}_{12}$ particle $(2.59 \AA)$ than in the $\mathrm{Fe}_{12} \mathrm{Pt}$ system $(2.48 \AA)$. However, both these distances are reduced comparing to the Fe-Pt distance in the bulk $(2.70 \AA)$. The NN distances in the shells are equal $d(\mathrm{Pt}-\mathrm{Pt})=2.72 \AA$ and $d(\mathrm{Fe}-\mathrm{Fe})=2.60 \AA$, respectively. The former one is reduced only by $2 \%$ comparing to the distances between Pt atoms in the fcc crystal $(2.77 \AA)$, while the latter is 


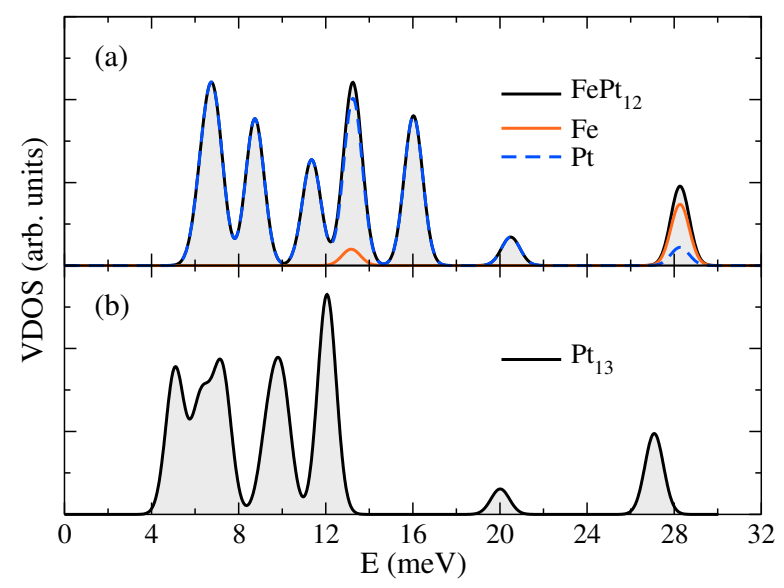

FIG. 9. Vibrational spectra of (a) $\mathrm{FePt}_{12}$ and (b) $\mathrm{Pt}_{13}$ icosahedra.

larger by $4.4 \%$ than between the Fe atoms in the bcc crystal $(2.49 \AA)$. As we show below, it has a strong impact on the stability of icosahedral particles.

The vibrational spectra of the smallest icosahedra are presented in Figs. 9 and 10. All these spectra are symmetric with respect to the $x, y, z$ components, therefore only the total and atom-projected DOS functions are presented. For $\mathrm{FePt}_{12}$, the spectrum consists of seven lines with a dominant contribution from $\mathrm{Pt}$ atoms. The Fe atom takes part only in two modes at 13.3 and $28.3 \mathrm{meV}$. The second highest mode at $20.5 \mathrm{meV}$ is the fully symmetric $A_{g}$ mode in which all $\mathrm{Pt}$ atoms vibrate along radial directions (breathing mode). All energies are real indicating dynamical stability of this system. This spectrum can be compared with the VDOS of the $\mathrm{Pt}_{13}$ icosahedron with all peaks shifted to lower energies [see Fig. 9(b)]. This shift is caused by larger interatomic Pt-Pt distances between the central atom and the shell atoms $(2.63 \AA)$ and between the atoms in the shell $(2.77 \AA)$. The latter equals to the NN distance in the bulk crystal. The highest mode dominated by the movement of the central $\mathrm{Pt}$ atom decreases its energy to $27.1 \mathrm{meV}$, while the breathing mode is shifted to $20 \mathrm{meV}$. The acoustic gap is reduced by about $2 \mathrm{meV}$.

In stark contrast, the spectrum of $\mathrm{Fe}_{12} \mathrm{Pt}$ shows a few imaginary $\mathrm{Fe}$ modes in the range between $32 i$ and $20 i \mathrm{meV}$ [Fig. 10a)]. They are induced by the overstretched $\mathrm{Fe}-\mathrm{Fe}$ distance, which strongly reduces the interatomic force constants, and the small Fe-Pt distances. The distortion induced by the soft modes removes the symmetry elements and lowers the total energy by $21 \mathrm{meV} /$ atom. The VDOS calculated without the symmetry constraints (with all atoms displaced) still shows a few soft modes demonstrating that the particle cannot be completely stabilized in this geometry. Replacing the central $\mathrm{Pt}$ atom with Fe does not stabilize the $\mathrm{Fe}_{13}$ icosahedron (VDOS is not shown). Although, the Fe-Fe distance in the shell $(2.48 \AA)$ is comparable with the value in the bulk crystal, the distance between the

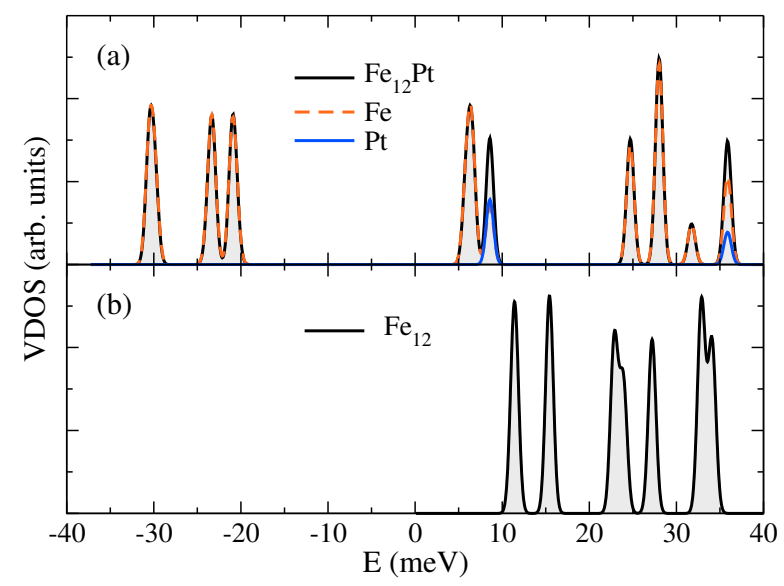

FIG. 10. Vibrational spectra of (a) $\mathrm{Fe}_{12} \mathrm{Pt}$ and (b) $\mathrm{Fe}_{12}$ icosahedra.

central atom and the shell is strongly reduced (2.36 $\AA$ ). Relaxation of the $\mathrm{Fe}_{13}$ icosahedron without the symmetry lowers the total energy by $63 \mathrm{meV} /$ atom, however, the VDOS calculated using displacements of all atoms still exhibits the imaginary modes. As shown by numerous test calculations, $\mathrm{Fe}_{12} \mathrm{Pt}$ or $\mathrm{Fe}_{13}$ icosahedra can be stabilized only by removing the central $\mathrm{Pt}$ or Fe atom and relaxing the positions of all the remaining Fe atoms. After relaxation, the radius of the Fe shell decreases to $2.28 \AA$, and the $\mathrm{Fe}-\mathrm{Fe}$ distance to $2.39 \AA$. It results in the shift of the lowest mode to $11.4 \mathrm{meV}$ largely increasing the acoustic gap, and the shift of the highest mode to the lower energy $34.3 \mathrm{meV}$ [see Fig. 10(b)].

$\mathbf{F e}_{12} \mathbf{P t}_{\mathbf{4 3}}$-The icosahedral particles with $N=55$ consists of a central atom and two shells with 12 and 42 atoms. In $\mathrm{Fe}_{12} \mathrm{Pt}_{43}$, the radius of the Fe shell equals to $2.59 \AA$ and is larger than in $\mathrm{Fe}_{12} \mathrm{Pt}$ due to the attraction from the $\mathrm{Pt}$ shell. In this outer shell, the $\mathrm{Pt}$ atoms are located in two distinct distances from the center: $5.05 \AA$ (corner atoms) and $4.48 \AA$ (edge atoms). The NN distances in the shells are $d(\mathrm{Fe}-\mathrm{Fe})=2.72 \AA$ and $d(\mathrm{Pt}-\mathrm{Pt})=2.66 \AA$. The VDOS of this particle is presented in Fig. 11(a). There are two soft modes with very close energies at $16.35 i \mathrm{meV}\left(E_{g}\right)$ and $16.33 i \mathrm{meV}\left(T_{g}\right) \mathrm{meV}$ of mainly $\mathrm{Fe}$ character. The origin of this soft mode is similar as in the $\mathrm{Fe}_{12} \mathrm{Pt}$ particle: enlarged $\mathrm{Fe}-\mathrm{Fe}$ distance in the Fe shell and reduced Fe-Pt distance. The energy vibrations of the outer $\mathrm{Pt}$ shell have a dominant contribution in the range between 4 and $24 \mathrm{meV}$ with some participation of Fe atoms. In this energy range, the central Pt atom contributes only to three modes. Two highest modes of mainly Fe character are separated by about $4 \mathrm{meV}$ and are located at $\sim 32$ and $\sim 36 \mathrm{meV}$. Interestingly, there is also small contribution of the central $\mathrm{Pt}$ atom in the highest mode.

We have used the soft modes to distort the particle and relax it to the lower symmetry $S_{2}$. The relaxation decreases the particle energy only by $1 \mathrm{meV} /$ atom and does not remove completely the soft modes from the VDOS. 


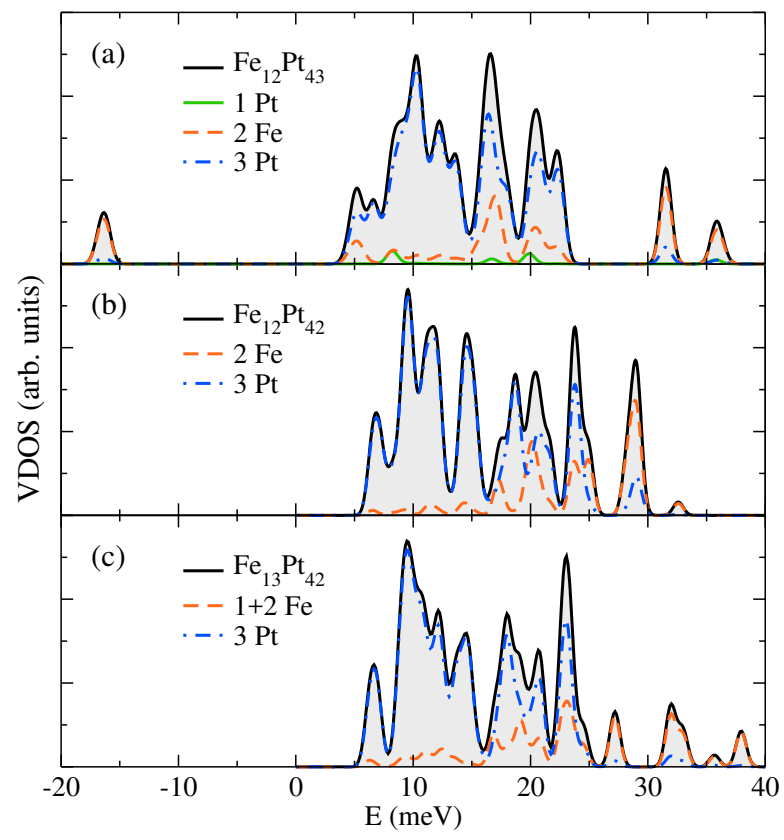

FIG. 11. Vibrational density of states of (a) $\mathrm{Fe}_{12} \mathrm{Pt}_{43}$, (b) $\mathrm{Fe}_{12} \mathrm{Pt}_{42}$, and (c) $\mathrm{Fe}_{13} \mathrm{Pt}_{42}$ icosahedra.

As in the smaller icosahedron, the particle can be stabilized by removing the central $\mathrm{Pt}$ atom, which leads to the two-shell system $\mathrm{Fe}_{12} \mathrm{Pt}_{42}$ with modified interatomic distances. All radial distances decrease; the radius of the Fe shell contracts to $2.48 \AA$ and the distances of the $\mathrm{Pt}$ atoms from the center are reduced to 4.99 and $4.46 \AA$. These changes not only stabilize the structure (removing the soft mode) but modify the whole spectrum of vibrations. Due to smaller Pt-Pt distances, the spectrum of $\mathrm{Pt}$ vibrations is shifted to higher energies. At the same time, the energies of two highest modes of mainly Fe character decrease to 29 and $32.6 \mathrm{meV}$ due to larger $\mathrm{Fe}-\mathrm{Pt}$ distances, thus the gap separating these modes is substantially reduced. The highest mode is the internal breathing mode of the Fe shell.

$\mathbf{F e}_{13} \mathbf{P t}_{\mathbf{4 2}}$-We have performed calculations for the similar core-shell system $\mathrm{Fe}_{13} \mathrm{Pt}_{42}$ with the central $\mathrm{Pt}$ atom replaced by the Fe atom. Due to the smaller atom in the centre, all interatomic distances are reduced comparing to the $\mathrm{Fe}_{12} \mathrm{Pt}_{43}$ system. The larger change is observed for the Fe core. The radius contracts to $2.53 \AA$ and the NN Fe-Fe distance to $2.66 \AA$. The NN distance in the Pt shell $(2.64 \AA)$ and the distances from the centre to corner $(5.02 \AA)$ and edge $(4.47 \AA) \mathrm{Pt}$ atoms are only slightly reduced and very similar to those in the NP without the central atom. The VDOS presented in Fig. 11(c) shows the dynamical stability of this system. Comparing to the $\mathrm{Fe}_{12} \mathrm{Pt}_{42}$ system, the energies of $\mathrm{Pt}$ vibrations are slightly extended to higher energies. The spectrum of $\mathrm{Fe}$ vibrations shows larger changes and the highest peak is shifted to $38 \mathrm{meV}$, above the values found in both similar systems.

$\mathbf{F e}_{\mathbf{4}} \mathbf{P t}_{\mathbf{1 2}}-$ The VDOS of the $\mathrm{Fe}_{43} \mathrm{Pt}_{12}$ system is pre-

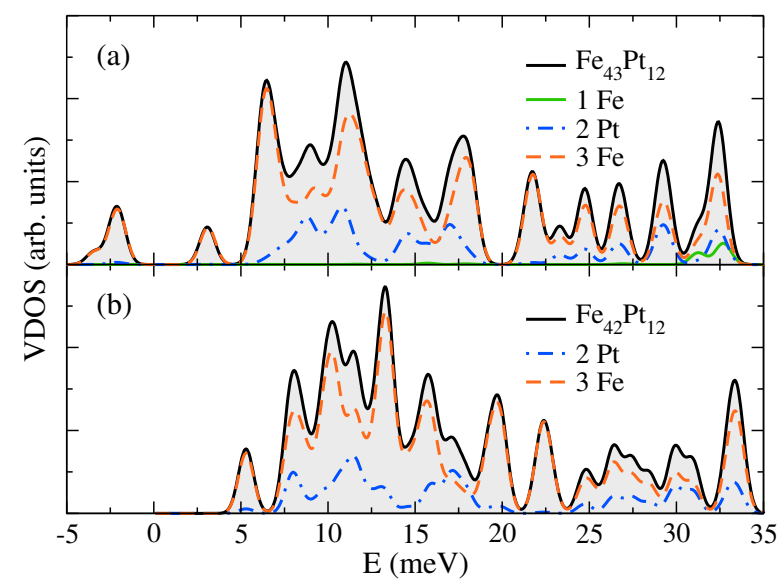

FIG. 12. Vibrational density of states of (a) $\mathrm{Fe}_{43} \mathrm{Pt}_{12}$ and (b) $\mathrm{Fe}_{42} \mathrm{Pt}_{12}$ icosahedra.

sented in Fig. 12(a). The optimized radial distances are equal to $2.64 \AA$ for the Pt shell, and 5.09 and $4.37 \AA$ for the Fe shell. The NN distances in the Pt and Fe shells equal 2.78 and $2.68 \AA$, respectively. Strongly elongated Fe-Fe distance leads to three unstable Fe modes at $3.45 i \mathrm{meV}\left(A_{g}\right), 2.27 i \mathrm{meV}\left(E_{u}\right)$, and $2.09 i \mathrm{meV}\left(T_{u}\right)$. The vibrations of $\mathrm{Fe}$ and $\mathrm{Pt}$ atoms are distributed over the whole energy spectrum $(2-34 \mathrm{meV})$ with the dominant contribution from the Fe surface shell. The central Fe atom is involved only in the highest modes at about $32 \mathrm{meV}$. We have repeated calculations without the central $\mathrm{Fe}$ atom obtaining the stable structure with all real vibrational energies presented in Fig. 12(b). The radii of both shells are slightly reduced to $2.60 \AA(\mathrm{Fe})$ and 5.05$4.36 \AA(\mathrm{Pt})$, which results in shortening of the NN distances $d(\mathrm{Pt}-\mathrm{Pt})=2.74$ and $d(\mathrm{Fe}-\mathrm{Fe})=2.65 \AA$. It stabilizes the particle and shifts the whole spectrum of vibrations to higher energies. The lowest mode at $5.3 \mathrm{meV}$, which defines the acoustic gap, and the highest mode at $33.6 \mathrm{meV}$ are mainly of the Fe character.

\section{Thermodynamic properties}

For the stable nanoalloys, the thermodynamic properties can be studied within the harmonic approximation. In Figs. 13 and 14 we have compared the vibrational heat capacity calculated for the NPs with different geometries and number of atoms in the temperature range $T=0-300 \mathrm{~K}$. The insets present the results below $T=30 \mathrm{~K}$. At low temperatures, the heat capacity of cuboctahedral particles presented in Fig. 13 decreases with the increasing number of atoms. It is connected with the relatively larger VDOS at lower energies in the smallest particles. This tendency is reversed around $T=50 \mathrm{~K}$. Since in the smaller particles, the energy cut-off is shifted to larger values, their heat capacity is reduced at higher temperatures comparing to larger particles. At each temperature, all systems have larger heat capacity than the 


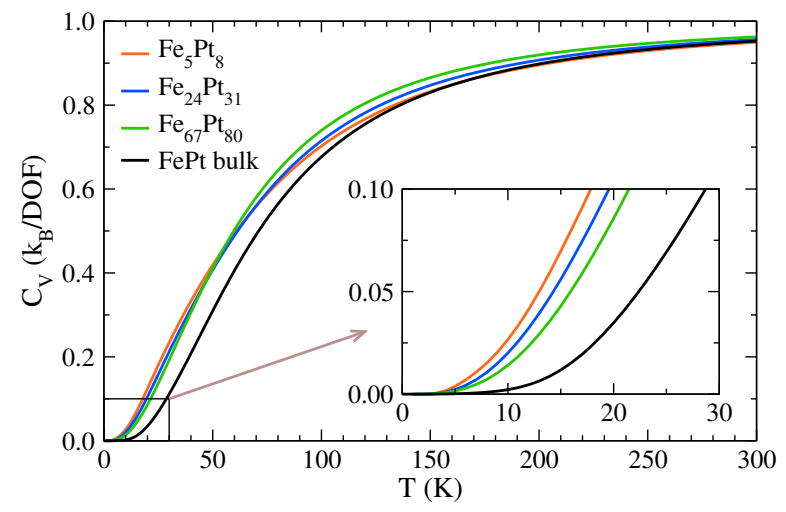

FIG. 13. Vibrational heat capacity for the cuboctahedral particles compared with the FePt crystal lattice heat capacity calculated per one degree of freedom (DOF).

bulk FePt crystal.

In Fig. 14, we have compared the heat capacity of two icosahedral particles $\mathrm{Fe}_{12} \mathrm{Pt}_{42}$ and $\mathrm{Fe}_{42} \mathrm{Pt}_{12}$ with one of the cuboctahedra $\mathrm{Fe}_{24} \mathrm{Pt}_{31}$. They have similar sizes and total numbers of atoms. At the lowest temperatures, the cuboctahedron has larger heat capacity than the icosahedra because of the larger acoustic gap in the icosahedra (5-6 meV) than in the cuboctahedron $(1 \mathrm{meV})$. Above around $60 \mathrm{~K}$, the heat capacity of the icosahedra exceeds the values for the cuboctahedron, which exhibits the higher cut-off of the vibrational energies. Interestingly, in spite of different concentration of $\mathrm{Fe}$ and $\mathrm{Pt}$ atoms, both icosahedral NPs have very similar heat capacities in the entire range of temperatures. It demonstrates that the thermodynamic properties of nanoalloys are to a large extend determined by the geometry. At the lowest temperatures, due to the smaller acoustic gap, the heat capacity in $\mathrm{Fe}_{42} \mathrm{Pt}_{12}$ is slightly larger than in $\mathrm{Fe}_{12} \mathrm{Pt}_{42}$.

In Table III the vibrational entropy calculated for the selected nanoparticles is compared with the entropy of the FePt crystal. The average entropy is very similar for all nanoparticles and it is approximately $20 \%$ larger than in the bulk. The contribution from $\mathrm{Fe}$ vibrations in the subsurface layers or in the particle's core is slightly enhanced (about 6\%), but it is notably increased by $44 \%$ in the surface shell of the $\mathrm{Fe}_{42} \mathrm{Pt}_{12}$ particle. It is consistent with the unstable character and low melting temperatures of particles with Fe surface layers [45. The entropy of $\mathrm{Pt}$ vibrations in the icosahedral NPs is very similar in the core and surface layers and it is larger by $7 \%$ comparing to the bulk. The larger increase by about $23 \%$ is found for the surface of the cuboctahedral $\mathrm{Fe}_{24} \mathrm{Pt}_{31}$ particle. It shows that cuboctahedral particles are less stable than the icosahedra with the Pt outer shells.

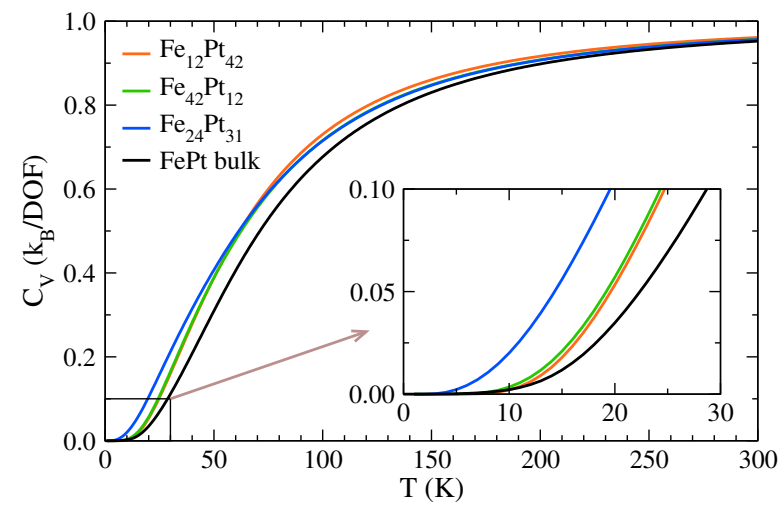

FIG. 14. Comparison of the vibrational heat capacity of two icosahedra with the cuboctahedral particle and the FePt crystal lattice heat capacity calculated per one degree of freedom (DOF).

TABLE II. Vibrational entropy calculated in units $k_{B}$ per one degree of freedom (DOF).

\begin{tabular}{|c|c|c|c|c|c|}
\hline & $\operatorname{FePt}\left(L 1_{0}\right)$ & $\mathrm{Fe}_{24} \mathrm{Pt}_{31}$ & $\mathrm{Fe}_{12} \mathrm{Pt}_{42}$ & $\mathrm{Fe}_{13} \mathrm{Pt}_{42}$ & $\mathrm{Fe}_{42} \mathrm{Pt}_{12}$ \\
\hline Total & 1.376 & 1.663 & 1.632 & 1.609 & 1.666 \\
\hline $\mathrm{Fe}$ & 1.144 & 1.246 & 1.204 & 1.216 & 1.646 \\
\hline $\mathrm{Pt}$ & 1.608 & 1.986 & 1.754 & 1.731 & 1.736 \\
\hline
\end{tabular}

\section{DISCUSSION AND SUMMARY}

The results obtained for all studied FePt systems have been summarized in Table【 The cuboctahedral NPs with the odd number of $\mathrm{Pt}$ layers stabilize in the distorted structure with the lower symmetry induced by the soft mode. In the systems with the even number of Pt layers such distortion is observed without breaking the $D_{4 h}$ symmetry. In the case of icosahedra with the perfect shell geometry, only the smallest particle $\mathrm{FePt}_{12}$ is dynamically stable. Other studied icosahedral systems are unstable due to the stretched Fe-Fe and reduced Fe-Pt distances and they can be stabilized by removing a central Fe or $\mathrm{Pt}$ atom or by replacing platinum by iron in the $\mathrm{Fe}_{12} \mathrm{Pt}_{43}$ nanoparticle. This result agrees with the previous observations that a large atom in the center of an icosahedron induces a large compressive stress and can destabilize the structure [56. The single-element icosahedra can be more stable without the central atoms [57, but in the core-shell particles with smaller atoms in the core, the central vacancy is not favored [58.

Both mechanisms of stabilization discussed here induce changes not only at the lowest vibrational energies but also at higher energies due to the modified interatomic distances. In particular, the deformations of the cuboctahedra with $\mathrm{N}=13$ and 55 induced by the soft modes lead to the appearance of the Fe modes with the energies higher than in the bulk FePt alloy 48 .

All these calculations are performed for the ground states corresponding to $T=0 \mathrm{~K}$. Thermal fluctuations 
may stabilize some of the considered systems, which exhibit the soft-mode behavior. Indeed, the ab initio MD studies demonstrate very high stability of the icosahedral particles with the Pt atoms in the outer shell [45. In these simulations performed at various temperatures, the $\mathrm{Fe}_{12} \mathrm{Pt}_{43}$ particle is very stable even at $T=1000 \mathrm{~K}$ and new preliminary studies indicate a very high melting temperature (around $1500 \mathrm{~K}$ ). It shows that the unstable behavior due to the presence of central atoms, may be relevant only at very low temperatures.

The MD simulations show that the particles with the Fe atoms in the outer shell are very unstable and strongly distorted even at low temperatures [45]. It is induced by a tendency of $\mathrm{Pt}$ atoms to move to the surface layer even in the platinum deficient particles. This is in perfect correspondence with the present study. Each of the three cuboctahedral NPs with the Fe atoms in the surface layers exhibits a few soft modes, which strongly distort their geometries. For none of these systems, we could obtain the dynamically stable structure and manage to calculate the vibrational spectrum. Two icosahedral particles with the Fe atoms in the outer shell were stabilized only by removing the central atom.
Similarly to other systems [19, 24, 34, the modified vibrational spectra of FePt nanoparticles influence their thermodynamic properties. The calculated heat capacities of nanoparticles are increased comparing to the lattice heat capacity of the crystal due to the enhanced VDOS at lower energies. At low temperatures, the heat capacity of cuboctahedral particles is larger than in icosahedra, which have larger acoustic gaps. Also, the vibrational entropy of nanoparticles is enhanced comparing to the bulk material. The largest increase of entropy is found for the Fe atoms in the outer shell of icosahedron, which confirms the unstable character of this system.

\section{ACKNOWLEDGMENTS}

The authors acknowledge support by the COST Action MP0903 "Nanoalloys as Advanced Materials: From Structure to Properties and Applications" and by the Polish National Science Center (NCN) under Project No. 2011/01/M/ST3/00738.
[1] R. Ferrando, J. Jellinek, and R. L. Johnston, Chem. Rev. 108, 845 (2008).

[2] R. L. Johnston, R. Ferrando, C. R. Henry, S. L. Horswell, B. F. G. Johnson, P. Lievens, Faraday Discussion, Nanoalloys:From theory to Applications, 138 (2008).

[3] M. M. Mariscal, O. A. Oviedo, and E. P. M. Leiva, Metal Clusters and Nanoalloys. From Modeling to Applications, Springer, New York, 2013.

[4] C. T. Campbell, Nature 432, 282 (2004); J. K. Norskov, T. Bligaard, J. Rossmeisl, C. H. Christensen, Nature. Chem. 1, 37 (2009).

[5] X. Huang, P. K. Jain, I. H. El-Sayed, M. A. El-Sayed, Nanomedicine 2, 681 (2007). L.-M. Lacroix, R. Bel Malaki, J. Carrey, S. Lachaize, G. F. Goya, B. Chaudret, M. Respaud, J. Appl. Phys. 105, 023911 (2009); S. Rose, A. Prevoteau, P. Elziere, D. Hourdet, Alba Marcellan and L. Leibler, Nature 505, 382 (2014).

[6] S. Sun, C. B. Murray, D. Weller, L. Folks, and A. Moser, Science 287, 1989 (2000).

[7] K. P. Bohnen and K. M. Ho, Surf. Sci. Rep. 19, 99 (1993).

[8] H. N. Frase, L. J. Nagel, J. L. Robertson, and B. Fultz, Philos. Mag. B 75, 335 (1997).

[9] B. Fultz, C. C. Ahn, E. E. Alp, W. Sturhahn, and T. S.Toellner, Phys. Rev. Lett. 79, 937 (1997).

[10] A. Kara and T.S. Rahman, Phys. Rev. Lett. 81, 1453 (1998).

[11] D. Y. Sun, X. G. Gong, and X. Q. Wang, Phys. Rev. B 63, 193412 (2001).

[12] R. Meyer, L. J. Lewis, S. Prakash, and P. Entel, Phys. Rev. B 68, 104303 (2003).

[13] A. Kara, A. N. Al-Rawi, and T. S Rahman, J. Comput. Theor. Nanosci. 1, 216 (2004).

[14] J. Łażewski, J. Korecki, and K. Parlinski, Phys. Rev. B 75, 054303 (2007).
[15] T. Ślezak, J. Łażewski, S. Stankov, K. Parlinski, R. Reitinger, M. Rennhofer, R. Rüffer, B. Sepiol, M. Ślezak, N. Spiridis, M. Zajac, A.I. Chumakov, and J. Korecki, Phys. Rev. Lett. 99, 066103 (2007).

[16] S. Stankov, R. Rohlsberger, T. Ślezak, M. Sladecek, B. Sepiol, G. Vogl, A.I. Chumakov, R. Rüffer, N. Spiridis, J. Łażewski, K. Parlinski, and J. Korecki, Phys. Rev. Lett. 99, 185501 (2007).

[17] J. Łażewski, P. Piekarz, A. M. Oleś, J. Korecki, and K. Parlinski, Phys. Rev. B 76, 205427 (2007).

[18] B. Roldan Cuenya, M. Alcántara Ortigoza, L. K. Ono, F. Behafarid, S. Mostafa, J. R. Croy, K. Paredis, G. Shafai, T. S. Rahman, L. Li, Z. Zhang, and J. C. Yang, Phys. Rev. B 84, 245438 (2011).

[19] G. Shafai, M. Alcántara Ortigoza, and T. S. Rahman, J. Phys.: Condens. Matter 24, 104026 (2012).

[20] N. Spiridis, M. Zajac, P. Piekarz, A.I. Chumakov, K. Freindl, J. Goniakowski, A. Kozioł-Rachwał, K. Parlinski, M. Ślezak, T. Ślezak, U.D. Wdowik, D. Wilgocka-Ślezak, and J. Korecki, Phys. Rev. Lett. 115, 186102 (2015).

[21] D. Bozyigit, N. Yazdani, M. Yarema, O. Yarema, W. M. M. Lin, S. Volk, K. Vuttivorakulchai, M. Luisier, F. Juranyi, and V. Wood, Nature 531, 618 (2016)

[22] A. Seiler, P. Piekarz, S. Ibrahimkutty, D. G. Merkel, O. Waller, R. Pradip, A. I. Chumakov, R. Ruffer, T. Baumbach, K. Parlinski, M. Fiederle, and S. Stankov, Phys. Rev. Lett. 117, 276101 (2016).

[23] H. Yildirim, A. Kara and T. S. Rahman, J. Phys.: Condens. Matter 21, 084220 (2009).

[24] D. P. Rojas, L. Fernández Barquín, J. Rodríguez Fernández, L. Rodríguez Fernández, J. Gonzalez, Nanotechnology 21, 445702 (2010).

[25] B. Roldan Cuenya, A. Naitabdi, J. Croy, W. Sturhahn, J. Y. Zhao, E. E. Alp, R. Meyer, D. Sudfeld, E. Schuster 
and W. Keune, Phys. Rev. B 76, 195422 (2007).

[26] B. Roldan Cuenya, L. K. Ono, J. R. Croy, A. Naitabdi, H. Heinrich, J. Zhao, E. E. Alp, W Sturhahn, and W. Keune, Appl. Phys. Lett. 95, 143103 (2009).

[27] B. Roldan Cuenya, J. R. Croy, L. K. Ono, A. Naitabdi, H. Heinrich, W. Keune, J. Y. Zhao, W. Sturhahn, E. E. Alp, M. Hu, Phys. Rev. B 80, 125412 (2009).

[28] P. Gruene, D. M. Rayner, B. Redlich, A. F. G. van der Meer, J. T. Lyon, G. Meijer, A. Fielicke, Science 321, 674 (2008).

[29] V. Juvé, A. Crut, P. Maioli, M. Pellarin, M. Broyer, N. Del Fatti, F. Vallée, Nano Lett. 10, 1853 (2010).

[30] H. E. Sauceda, D. Mongin, P. Maioli, A. Crut, M. Pellarin, N. Fatti, F. Vallée, and I. L. Garzón, J. Phys. Chem. C 116, 25147 (2012).

[31] H. Portales, N. Goubert, L. Saviot, S. Adichtchev, D. B. Murray, A. Mermet, E. Duval, M.-P. Pileni, Proc. Natl. Acad. Sci. 105, 14784 (2008).

[32] M. Bayle, N. Combe, N. M. Sangeetha, G. Viau, R. Carles, Nanoscale 6, 9157 (2014).

[33] M. Bayle, P. Benzo, N. Combe, C. Gatel, C. Bonafos, G. Benassayag, R. Carles, Phys. Rev. B 89, 195402 (2014).

[34] R. Carles, P. Benzo, B. Pécassou, end C. Bonafos, Sci. Rep. 6, 39164 (2016).

[35] L. Graf and A. Kussmann, Z. Phys. 36, 544 (1935).

[36] Y. Tamada, S. Yamamoto, M. Takano, S. Nasu, and T. Ono, Appl. Phys. Lett. 90, 162509 (2007).

[37] Y. Tamada, S. Yamamoto, S. Nasu, and T. Ono, Phys. Rev. B 78, 214428 (2008).

[38] R. M. Wang, O. Dmitrieva, M. Farle, G. Dumpich, H. Q. Ye, H. Poppa, R. Kilaas, and C. Kisielowski, Phys. Rev. Lett. 100, 017205 (2008).

[39] A. Fortunelli and A. M. Velasco, J. Mol. Struct. (Theochem) 487, 251 (1999).

[40] R. V. Chepulskii, J. Velev, and W. H. Butler, J. Appl. Phys. 97, 10J311 (2005).

[41] M. E. Gruner, G. Rollmann, P. Entel, and M. Farle, Phys. Rev. Lett. 100, 087203 (2008).

[42] M. E. Gruner, J. Phys.: Conf. Ser. 200, 072039 (2010).
[43] M. E. Gruner and P. Entel, Beilstein J. Nanotechnol. 2, 162 (2011).

[44] A. Kabir, J. Hu, V. Turkowski, R. Wu, R. Camley, and T. S. Rahman, Phys. Rev. B 92, 054424 (2015).

[45] P. T. Jochym, J. Łażewski, M. Sternik, and P. Piekarz, Phys. Chem. Chem. Phys. 17, 28096 (2015).

[46] Y. Tamada, R. Masuda, A. Togo, S. Yamamoto, Y. Yoda, I. Tanaka, M. Seto, S. Nasu, and T. Ono, Phys. Rev. B 81, 132302 (2010).

[47] S. Couet, M. Sternik, B. Laenens, A. Siegel, K. Parlinski, N. Planckaert, F. Gröstlinger, A. I. Chumakov, R. Rüffer, B. Sepiol, K. Temst, and A. Vantomme, Phys. Rev. B 82 094109 (2010).

[48] M. Sternik, S. Couet, J. Łażewski, P. T. Jochym, K. Parlinski, A. Vantomme, K. Temst, and P. Piekarz, J. Alloys and Comp. 651528 (2015).

[49] G. Kresse and J. Furthmüller, Phys. Rev. B 54, 11169 (1996); Comput. Mater. Sci. 6, 15 (1996).

[50] J. P. Perdew, K. Burke, and M. Ernzerhof, Phys. Rev. Lett. 77, 3865 (1996).

[51] P. E. Blöchl, Phys. Rev. B 50, 17953 (1994); G. Kresse and D. Joubert, ibid. 59, 1758 (1999).

[52] K. Parlinski, Z. Q. Li, and Y. Kawazoe, Phys. Rev. Lett. 78, 4063 (1997).

[53] K. Parlinski, Phonon Software, Kraków, 2013.

[54] See Supplemental Material at [URL will be inserted by publisher] for the electronic structure of the cuboctahedral nanoparticles and the animations of the soft modes.

[55] J. Łażewski, P. Piekarz, A. M. Oleś, and K. Parlinski, Phys. Rev. B 74, 174304 (2006).

[56] D. Bochicchio and R. Ferrando, Phys. Rev. B 87, 165435 (2013).

[57] C. Mottet, G. Treglia, and B. Legrand, Surf. Sci. 383, L719 (1997).

[58] K. Laasonen, E. Panizon, D. Bochicchio, and R. Ferrando, J. Phys. Chem. C 117, 26405 (2013).

[59] K. Momma and F. Izumi, J. Appl. Crystallogr. 44, 1272 (2011). 